\title{
Viscoelastic Properties of Immiscible Polymer Blends under Steady and Transient Shear Flows
}

\author{
Yoshiaki TaKahashi, Shinichi Kitade, Naoki Kurashima, \\ and Ichiro NoDA \\ Department of Applied Chemistry, Nagoya University, \\ Nagoya 464-01, Japan
}

(Received February 14, 1994)

\begin{abstract}
Viscoelastic properties of binary blends of immiscible polymers with almost the same viscosity were studied under steady and transient shear flows in the light of the scaling relations in the constitutive equation of textured materials presented by Doi and Ohta. Samples used were blends of polydimethylsiloxane (PDMS) and polybutadiene (PB) with the composition ratios of $3: 7,1: 1,7: 3$, and 9:1 for PDMS:PB by weight. It was found that both shear stress $\sigma_{12}$ and primary normal stress difference $N_{1}$ at steady state are proportional to shear rate $\dot{\gamma}$ except $N_{1}$ of $9: 1$ blend, and the rescaled transient stresses after stepwise increase of shear rate compose a single line in terms of strains if the step ratios of shear rates are constant, in accordance with the scaling relations. For the $9: 1$ blend, in which the contribution of $N_{1}$ of component polymers cannot be neglected because the interface is small, however, the extra primary normal stress difference at steady state after subtracting $N_{1}$ of component polymers from the measured one was found to be proportional to $\dot{\gamma}$ in accordance with the scaling relations.

KEY WORDS Immiscible Polymer Blends / Textured Materials /Scaling Relation / Shear Stress / Primary Normal Stress Difference /
\end{abstract}

Viscoelastic properties of immiscible polymer blends have been studied for a long time. ${ }^{1}$ In these blends, there exist various domain structures of component polymers which depend on composition, preparation method of the blends, and so on. Therefore, the viscoelastic properties of immiscible polymer blends are quite different from one system to others.

Moreover, the viscoelastic properties of immiscible polymer blends are different in flow modes used in the measurements. ${ }^{1,2}$ When they are measured under oscillatory flows with appropriate frequency and strain, the domain structures remain unchanged, so that the measured data can be used to characterize the structures. Under other flows, such as steady shear flow, the domains can be deformed, ruptured and aggregated by the flows, and hence the viscoelastic properties become complicated.

The relationship between the change of the domain structures by flows and the resulting viscoelastic properties is very interesting and also important in industries such as polymer processing. Despite of extensive work, however, the fundamental relationship between structures and viscoelastic properties has not be elucidated since there was no appropriate model or theory for such systems.

Recently, Doi and $\mathrm{Ohta}^{3,4}$ presented a theory of rheology for the $1: 1$ mixtures of two immiscible Newtonian fluids with the same viscosity and density which have complex domain structures called "texture" under flow. Taking into account time evolution of the area and orientation of interface of the fluids under flow field and interfacial tension opposing to the deformation, they predicted interesting scaling properties. 
According to their theory, shear stress $\sigma_{12}$ and primary normal stress difference $N_{1}$ under steady shear flows are predicted to be proportional to shear rate $\dot{\gamma}$.

$$
\begin{gathered}
\sigma_{12} \propto \dot{\gamma} \\
N_{1} \propto|\dot{\gamma}|
\end{gathered}
$$

Another application of the scaling relation is proposed for transient stress when the shear rate is changed from an initial one $\dot{\gamma}_{1}$ to a final one $\dot{\gamma}_{\mathrm{F}}$ at time $t=0$. In this case, the transient stresses $\sigma\left(t, \dot{\gamma}_{\mathrm{I}}, \dot{\gamma}_{\mathrm{F}}\right)$ divided by the initial steady stress $\sigma\left(\dot{\gamma}_{\mathrm{I}}\right)$ compose a single curve in terms of strain $\dot{\gamma}_{\mathrm{F}} t$, irrespective of shear rate, if the shear rate ratio $\dot{\gamma}_{F} \dot{\gamma}_{I}$ is maintained constant.

$$
\sigma\left(t, \dot{\gamma}_{\mathrm{I}}, \dot{\gamma}_{\mathrm{F}}\right) / \sigma\left(\dot{\gamma}_{\mathrm{I}}\right)=f\left(\dot{\gamma}_{\mathrm{F}} t, \dot{\gamma}_{\mathrm{F}} \dot{\gamma}_{\mathrm{I}}\right)
$$

The above scaling relations were first observed for liquid crystalline polymers ${ }^{5}$ in which the origin of stress are quite different from that in binary mixtures of immiscible fluids. ${ }^{6}$

In a previous work, ${ }^{7}$ we measured viscoelastic properties of binary mixtures of immiscible Newtonian fluids having almost the same viscosity over a wide range of composition and reported that the above scaling relationship holds not only for $1: 1$ blends but also over a wide range of composition. These results suggest that the scaling relations are applicable to a wide variety of systems which have textured structures.

The purpose of this work is to study the viscoelastic properties of binary blends of immiscible polymers, both of which are viscoelastic fluids having almost the same viscosity, in the light of the scaling relations of textured materials. Here, we measured stresses under steady shear flows and after the step increase of shear rate at several compositions.

\section{EXPERIMENTAL}

Two polymer samples used as components of blends were a polydimethylsiloxane (PDMS)
KF96H-100000 of Shinetsu Chemical Co. and a polybutadiene (PB) of Scientific Polymer Products. The PB sample was fractionated a few times from benzene/methanol solutions. Nominal molecular weight of PDMS is $1 \times 10^{5}$ and $M_{w}$ and $M_{w} / M_{n}$ of fractionated PB determined by GPC using polystyrene calibration curve are $1.7_{6} \times 10^{4}$ and $1.4_{7}$, respectively. The shear stress $\sigma_{12}$ and primary normal stress difference $N_{1}$ of these polymers are proportional to the first and second order of shear rate $\dot{\gamma}$, respectively, up to the highest shear rate measured in this work, indicating a typical viscoelastic behavior of molten polymers in the linear region of deformation. It should be noted that $N_{1}$ was detectable only at high shear rates, as shown later. The viscosities and densities of PDMS and PB at $20^{\circ} \mathrm{C}$ are $107 \mathrm{~Pa} \mathrm{~s}^{-1}$ and $0.977 \mathrm{~g} \mathrm{~cm}^{-3}$, and $97.8 \mathrm{~Pa} \mathrm{~s}^{-1}$ and $0.907 \mathrm{~g} \mathrm{~cm}^{-3}$, respectively.

Polymer mixtures with certain compositions were gently stirred to become milky in a vessel for a few minutes with a spatula. Then they were kept in vacuo for about 10 minutes to get bubble-free blend samples. The blending ratios of PDMS : PB were $3: 7,1: 1,7: 3$, and $9: 1$ by weight.

Viscoelastic measurements were carried out with a Mechanical Spectrometer RMS800 of Rheometrics Co. by using the cone-plate geometry with $5 \mathrm{~cm}$ diameter and 0.04 radian cone-angle. Shear stress and primary normal stress difference under steady shear flows and after step increase of shear rate were measured. All the measurements were carried out at room temperatures $\left(20-23^{\circ} \mathrm{C}\right)$. Details of the measurements are practically the same as those for immiscible Newtonian fluid mixtures reported previously. ${ }^{7}$

\section{RESULTS}

Figure 1 shows double logarithmic plots of $\sigma_{12}$ against $\dot{\gamma}$ for all the blend samples. Data for component polymers are also shown for comparison. It is clear that the $\sigma_{12}$ data of 


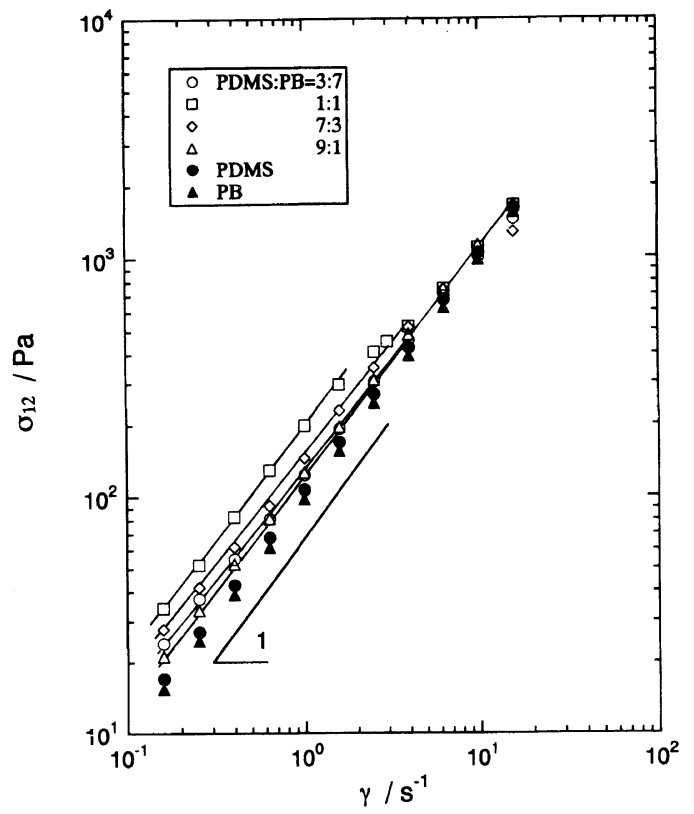

Figure 1. Double logarithmic plots of shear stress $\sigma_{12}$ for blend samples against shear rate $\dot{\gamma}$. The data for component polymers are also shown for comparison. Symbols are denoted in the figure.

blends are always higher than those of component polymers and $\sigma_{12}$ becomes the highest for $1: 1$ blend. The data for each blend sample can be represented by the respective straight line at the shear rate lower than a critical one $\dot{\gamma}^{*}$. The slopes evaluated by the least-square method for $3: 7,1: 1,7: 3$, and $9: 1$ blends are $0.92,0.95,0.92$, and 0.96 , respectively, slightly lower than, but close to the prediction of eq 1a. At the shear rates higher than $j^{*}, \sigma_{12}$ starts to deviate from the respective straight line and shows somewhat weaker shear rate dependence. The more the composition of the blend deviates from $50 \%$, the higher $\dot{\gamma}^{*}$ is. The deviation was not observed for $9: 1$ blend.

Figure 2 shows double logarithmic plots of $N_{1}$ against $\dot{\gamma}$ for all the blend samples. Data for component polymers are also shown for comparison. In similarity to $\sigma_{12}$, the $N_{1}$ data for blends are always higher than those of component polymers and $N_{1}$ becomes the highest for $1: 1$ blend. The data for $7: 3$ and

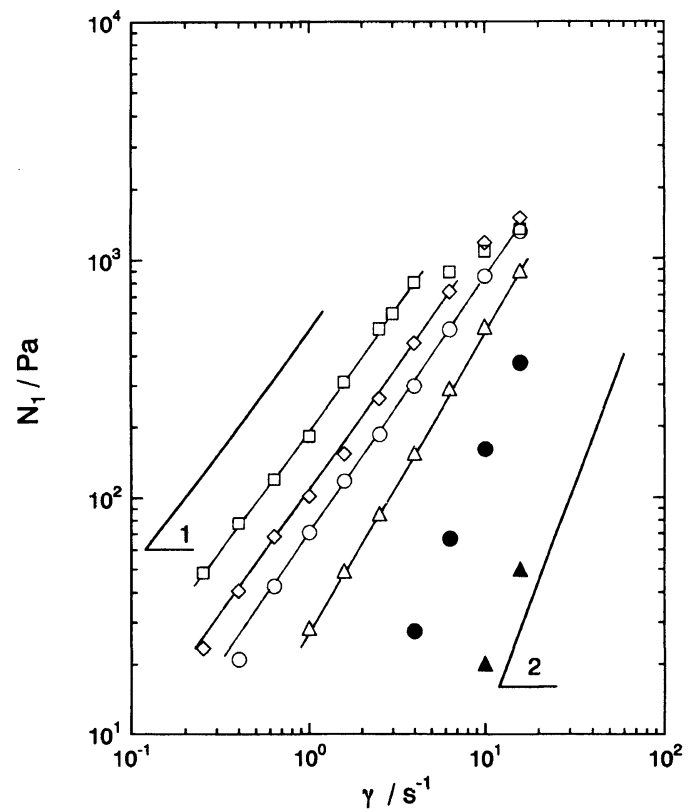

Figure 2. Double logarithmic plots of primary normal stress difference $N_{1}$ for blend samples against shear rate $\dot{\gamma}$. The data for component polymers are also shown for comparison. Symbols are the same as those in Figure 1.

$1: 1$ blend sample can be also represented by the respective straight line at $\dot{\gamma}<\dot{\gamma}^{*}$ and deviate from the line at $\dot{\gamma}>\dot{\gamma}^{*}$, though the values of $\dot{\gamma}^{*}$ for $N_{1}$ are slightly higher than those for $\sigma_{12}$. The deviation is not observed for $3: 7$ and 9:1 blends. The slopes of the straight lines evaluated by the least square method for $3: 7$, $1: 1$, and $7: 3$ blends are 1.07, 1.02, and 1.05, respectively, slightly higher than, but close to the prediction of eq $1 \mathrm{~b}$. However, the slope for 9:1 blend evaluated by the least square method was 1.27 , which is significantly higher than the slopes for other blend samples, but still lower than the slopes for component polymers. The discrepancy of the predicted and observed slopes for 9:1 blend will be discussed later.

As mentioned above, the slopes of $\sigma_{12}$ and $N_{1}$ are not exactly equal to 1 , but almost the same results were observed for Newtonian fluid mixtures. ${ }^{7}$ Therefore, we conclude that the scaling relations (eq 1) hold for blend samples of which compositions are not far from 1:1 


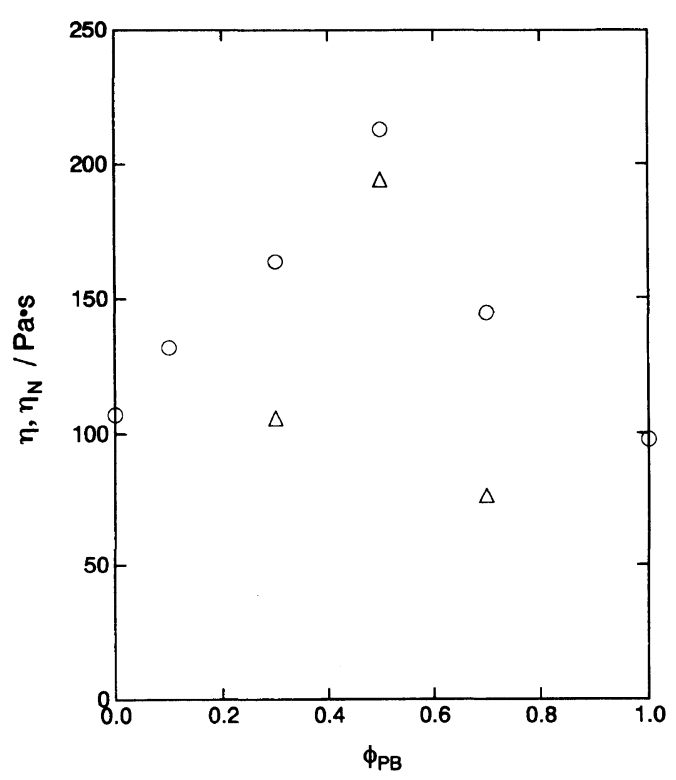

Figure 3. Composition dependence of shear viscosity $\eta$ and normal viscosity $\eta_{\mathrm{n}}$ for blend samples. Circles and triangles denote $\eta$ and $\eta_{\mathrm{n}}$, respectively.

over a wide range of shear rate at the same level as the Newtonian fluid mixtures. Disregarding the small deviations from unity, we evaluated shear viscosity $\eta\left(=\sigma_{12} / \dot{\gamma}\right)$ and normal viscosity ${ }^{4,7} \quad \eta_{\mathrm{n}}\left(=N_{1} / \dot{\gamma}\right)$ as a simple average of the data at $\dot{\gamma}<\dot{\gamma}^{*}$, for all the samples except $\eta_{\mathrm{n}}$ of $9: 1$ blend. Figure 3 shows composition dependences of $\eta$ and $\eta_{\mathrm{n}}$. Both $\eta$ and $\eta_{\mathrm{n}}$ have a maximum near $1: 1$ composition in similarity to Newtonian fluid mixtures. ${ }^{7} \eta$ is always higher than $\eta_{\mathrm{n}}$ and the difference becomes larger as the composition deviates from $50 \%$.

Figure 4 shows examples of the initial parts of transient shear stress after the stepwise increase of shear rate. The ratio of the final shear rate $\dot{\gamma}_{F}$ to the initial shear rate $\dot{\gamma}_{I}, \dot{\gamma}_{F} \dot{\gamma}_{I}$ was fixed at 2 for all these data. In Figure 5, the data in Figure 4 are replotted in the form of rescaled shear stress $\sigma\left(t, \dot{\gamma}_{\mathrm{I}}, \dot{\gamma}_{\mathrm{F}}\right) / \sigma\left(\dot{\gamma}_{\mathrm{I}}\right)$ against strain $\dot{\gamma}_{\mathrm{F}} t$. The plots of rescaled shear stress against strain for other blend samples at the shear rate ratio $\dot{\gamma}_{\mathrm{F}} / \dot{\gamma}_{\mathrm{I}}=2$ are also shown in this figure, where the data are vertically shifted to

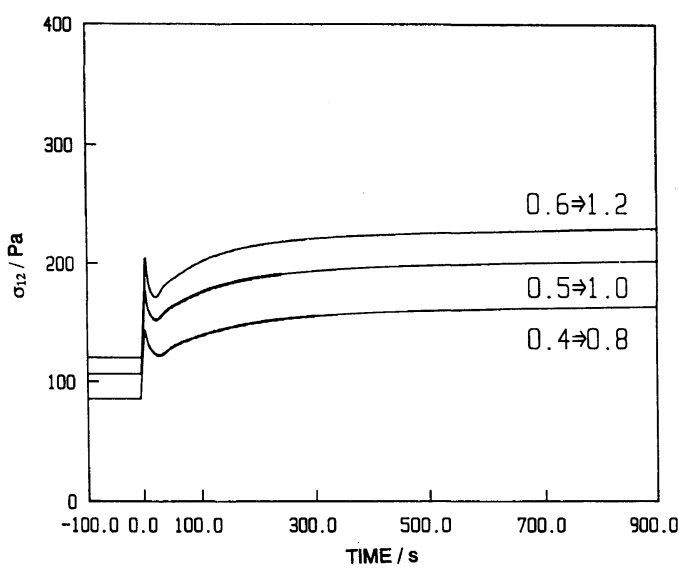

Figure 4. Examples of transient shear stress after step increase of shear rate. Sample is the $1: 1$ blend. Shear rates are denoted in the figure.

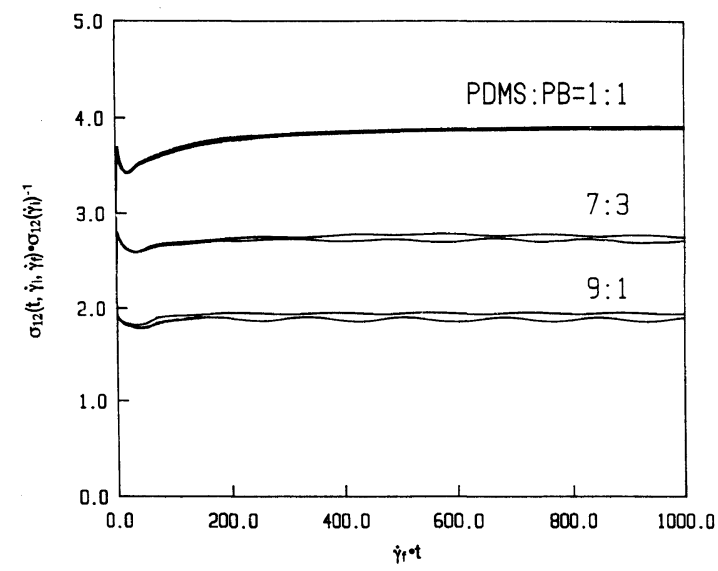

Figure 5. Plots of rescaled shear stress against strain at fixed shear rate ratio $\left(\dot{\gamma}_{\mathrm{F}} / \dot{\gamma}_{\mathrm{I}}=2\right)$. Composition of the samples are denoted in the figure. The data for $1: 1$ blend and $7: 3$ blend are vertically shifted by +2 and +1 , respectively.

avoid overlapping. The data for 3:7 blend are not shown since they are very similar to those of $7: 3$ blend. It can be seen that the data at each composition compose a single line. The feature of transient shear stress curves is the same as that of immiscible Newtonian fluid mixtures. ${ }^{7}$ The shear stress shows an undershoot and then gradually increases to a steady state value. The undershoot becomes shallower as the composition deviates from the value 


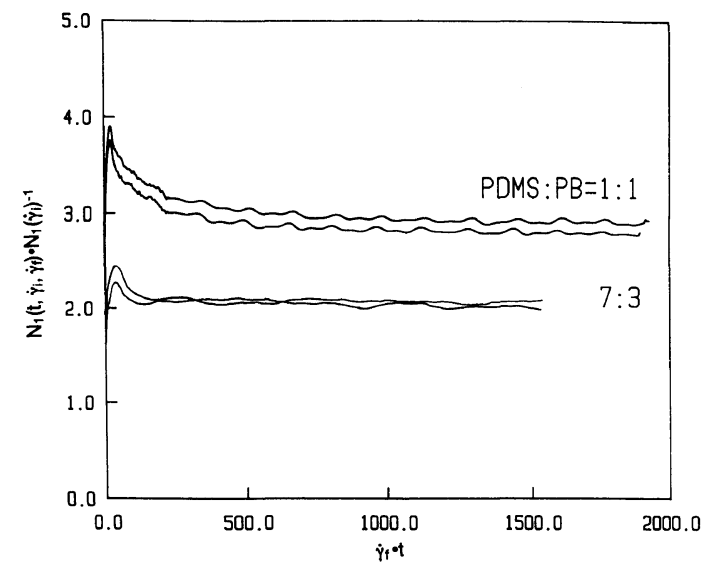

Figure 6. Plots of rescaled primary normal stress difference against strain at fixed shear rate ratio $\left(\dot{\gamma}_{F} / \dot{\gamma}_{I}=2\right)$. Composition of the samples are denoted in the figure. The data for $1: 1$ blend are vertically shifted by +1 .

where the maximum in viscosity is observed.

Figure 6 shows plots of rescaled normal stress difference against strain for $1: 1$ and $7: 3$ blends at the shear rate ratio $\dot{\gamma}_{\mathrm{F}} / \dot{\gamma}_{\mathrm{I}}=2$. The data are vertically shifted to avoid overlapping. It can be seen that the data at each composition compose a single line, though the data are somewhat scattered than those of $\sigma_{12}$ in Figure 5 since the measured $N_{1}$ is smaller than $\sigma_{12}$ and the experimental error in $N_{1}$ measurements is larger than that in $\sigma_{12}$. The shapes of rescaled $N_{1}$ curves are also similar to those of immiscible Newtonian fluid mixtures. ${ }^{7}$ There exist an overshoot and then gradually decrease to a steady state value. The data for 9:1 blend cannot be rescaled though the shape is similar to those of other blends, since the shear rate dependence at steady state is stronger than the first order of $\dot{\gamma}$.

\section{DISCUSSIONS}

Except for the primary normal stress difference data of $9: 1$ blend, all the data including the shear stress data of $9: 1$ blend are similar to those of immiscible Newtonian fluid mixtures. ${ }^{7}$ The steady state stresses are proportional to shear rate and the transient stresses after stepwise increase of shear rate can be rescaled, in accordance with the scaling relations, eq 1 and 2.

The measured stress is consist of two contributions from component polymers and interfaces. Strictly speaking, the scaling relations should hold for extra stresses due to the interfacial tension. ${ }^{3,4}$ As can be seen in Figures 1 and 2, viscoelastic properties of component polymers are in the linear region in the experimental range. Therefore, $\sigma_{12}$ and $N_{1}$ of components under steady shear flow can be expressed as

$$
\begin{gathered}
\sigma_{12}(\dot{\gamma})=\eta \dot{\gamma} \\
N_{1}(\dot{\gamma})=\psi_{12} \dot{\gamma}^{2}
\end{gathered}
$$

where $\psi_{12}$ is the primary normal stress coefficient. If we assume that the two contributions are additive, we may evaluate the extra shear stress $\Delta \sigma_{12}$ and extra primary normal stress difference $\Delta N_{1}$ due to the interfacial tention by subtracting the stresses of components from the observed ones.

$$
\begin{gathered}
\Delta \sigma_{12}(\dot{\gamma})=\sigma_{12}(\dot{\gamma})-\phi_{\mathrm{PDMS}}(\eta \dot{\gamma})_{\mathrm{PDMS}}-\phi_{\mathrm{PB}}(\eta \dot{\gamma})_{\mathrm{PB}} \\
\Delta N_{1}(\dot{\gamma})=N_{1}(\dot{\gamma})-\phi_{\mathrm{PDMS}}\left(\psi_{12} \dot{\gamma}^{2}\right)_{\mathrm{PDMS}} \\
-\phi_{\mathrm{PB}}\left(\psi_{12} \dot{\gamma}^{2}\right)_{\mathrm{PB}}
\end{gathered}
$$

where $\phi_{\mathrm{PDMS}}$ and $\phi_{\mathrm{PB}}$ are the volume fractions of PDMS and PB, respectively.

In immiscible blends, generally, the shear rates in domains are different from the applied value owing to interfacial tention, even if the viscosities of components are the same. At around 1:1 composition, however, the extra stresses are large enough to neglect the errors due to the difference in shear rates. When the composition is far from $1: 1$, on the other hand, the contribution of components comes from the larger domain or the matrix in which the shear rate is very close to the applied value. Thus, eq 6 and 7 may give a good approximation for the extra stresses at all the composition. 


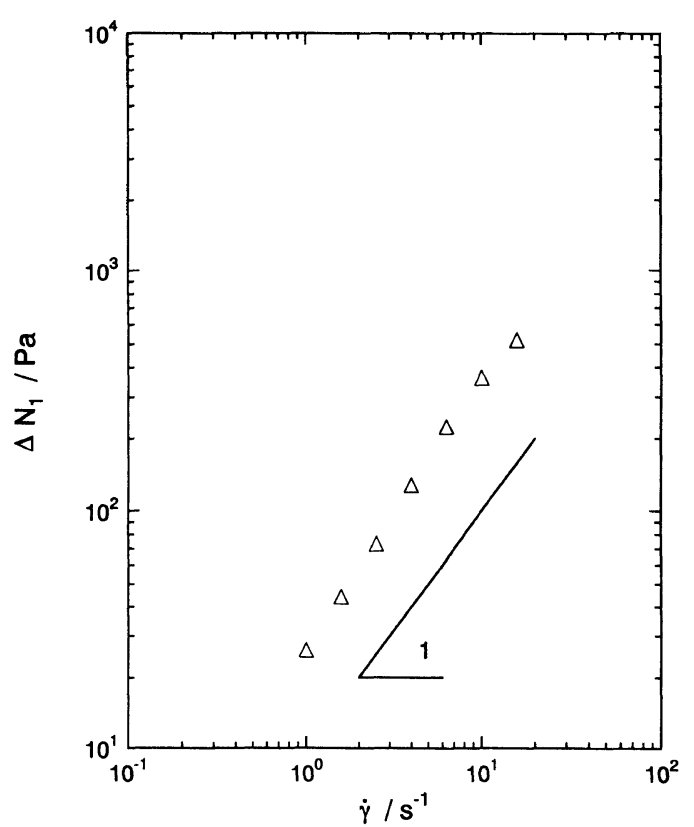

Figure 7. Double logarithmic plots of excess primary normal stress difference $\Delta N_{1}$ for $9: 1$ blend against shear rate $\dot{\gamma}$.

As shown in Figure 1, $\sigma_{12}$ of component polymers is not negligible compared to the measured $\sigma_{12}$ but it is proportional to $\dot{\gamma}$ at $\dot{\gamma}<\dot{\gamma}^{*}$ so that $\Delta \sigma_{12}$ is consistent with eq la at all the composition when $\dot{\gamma}<\dot{\gamma}^{*}$. As can be seen in Figure 2, moreover, $N_{1}$ of component polymers is almost negligible at most of compositions so that the observed $N_{1}$ can be regarded as $\Delta N_{1}$, which is consistent with eq 2a. At 9:1 composition, however, $N_{1}$ of component polymers is not negligible so that the observed shear rate dependence of $N_{1}$ becomes higher than unity at this composition.

Figure 7 shows double logarithmic plots of $\Delta N_{1}$ calculated by eq $7 v s . \dot{\gamma}$ for 9: 1 blend. It should be noted that $\phi_{\mathrm{PB}}\left(\psi_{12} \dot{\gamma}^{2}\right)_{\mathrm{PB}}$ is negligible compared to $\phi_{\text {PDMS }}\left(\psi_{12} \dot{\gamma}^{2}\right)_{\text {PDMS }}$. Apparently, $\Delta N_{1}$ is almost proportional to $\dot{\gamma}$, indicating that the scaling relation, eq $1 \mathrm{~b}$ holds for the extra primary normal stress difference.

The composition dependences of extra shear viscosity $\Delta \eta\left(=\Delta \sigma_{12} / \dot{\gamma}\right)$ and normal viscosity $\Delta \eta_{\mathrm{n}}\left(=\Delta N_{1} / \dot{\gamma}\right)$ for all samples including $9: 1$

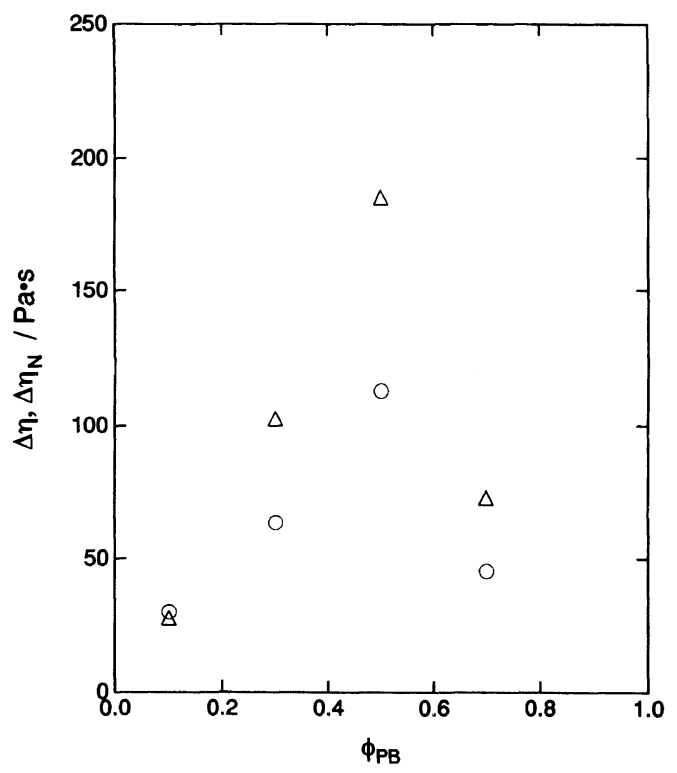

Figure 8. Composition dependence of extra shear viscosity $\Delta \eta$ and extra normal viscosity $\Delta \eta_{\mathrm{n}}$ for blend samples. Circles and triangles denote $\Delta \eta$ and $\Delta \eta_{\mathbf{n}}$, respectively.

blend are shown in Figure 8. It should be noted that $\Delta \eta_{\mathrm{n}}$ for $9: 1$ blend is consistent with other data in which the contribution of component polymers can be neglected and almost equal to $\eta_{\mathbf{n}}$ in Figure 3.

At transient state, the scaling relation, eq 2 should also hold for extra shear and normal stresses, i.e.,

$$
\frac{\Delta \sigma\left(t, \dot{\gamma}_{\mathrm{I}}, \dot{\gamma}_{\mathrm{F}}\right)}{\Delta \sigma\left(\dot{\gamma}_{\mathrm{I}}\right)}=f\left(\dot{\gamma}_{\mathrm{F}} t, \dot{\gamma}_{\mathrm{F}} \dot{\gamma}_{\mathrm{I}}\right)
$$

In our system, the viscosities of both components are very close so that we can assume that both are represented by $\eta$. Moreover, the shear stress reaches the steady value instantaneously when shear rate is changed, because of short relaxation time. For shear stress, therefore, eq 6 can be written as

$$
\begin{aligned}
\frac{\Delta \sigma_{12}\left(t, \dot{\gamma}_{\mathrm{I}}, \dot{\gamma}_{\mathrm{F}}\right)}{\Delta \sigma_{12}\left(\dot{\gamma}_{\mathrm{I}}\right)} & =\frac{\sigma_{12}\left(t, \dot{\gamma}_{\mathrm{I}}, \dot{\gamma}_{\mathrm{F}}\right)-\eta \dot{\gamma}_{\mathrm{F}}}{\sigma_{12}\left(\dot{\gamma}_{\mathrm{I}}\right)-\eta \dot{\gamma}_{\mathrm{I}}} \\
& =b \frac{\sigma_{12}\left(t, \dot{\gamma}_{\mathrm{I}}, \dot{\gamma}_{\mathrm{F}}\right)}{\sigma_{12}\left(\dot{\gamma}_{\mathrm{I}}\right)}-\frac{k}{a}
\end{aligned}
$$


where $k=\dot{\gamma}_{\mathrm{F}} / \dot{\gamma}_{\mathrm{I}}, \quad a=\Delta \eta / \eta, \quad \Delta \eta=\sigma_{12}\left(\dot{\gamma}_{\mathrm{I}}\right) / \dot{\gamma}_{\mathrm{I}}-\eta$ and $b=(a+1) / a$.

Since $k$ and $a$ are constant for each set of data, we can discuss extra transient shear stress in terms of $\sigma_{12}\left(t, \dot{\gamma}_{\mathrm{l}}, \dot{\gamma}_{\mathrm{F}}\right) / \sigma_{12}\left(\dot{\gamma}_{\mathrm{I}}\right)$, as shown in Figure 5 and also as used in a previous paper. From the results in Figure 5, we can conclude that the scaling relation is valid for extra transient shear stress after step change of shear rate.

For primary normal stress difference, eq 8 cannot be rewritten as for shear stress (eq 9) since $N_{1}$ of component polymers are proportional to $\dot{\gamma}^{2}$ and a numerical analysis is needed. As already mentioned, the contribution of component polymers are almost negligible except for $9: 1$ blend in the primary normal stress difference, so that the rescaled data in Figure 6 can be regarded as rescaled extra normal stress difference. For 9:1 blend, the numerical analysis is needed, but it could not be carried out in this work because of relatively large experimental error in excess transient normal stress difference. Considering the facts that scaling relation also holds for $\Delta N_{1}$ at steady states for 9:1 blend and the shape of transient data are similar to others, however, we may conclude that scaling relation is valid for the extra transient normal stress difference after step change of shear rate.

From the above results, we conclude that the scaling relations for the textured materials also hold for the excess stresses of immiscible polymer blends made of the component polymers with the same viscosity in a wide range of composition, if the shear rate is lower than a certain critical shear rate.

The shapes of transient stresses of polymer blends studied here are very similar to those of immiscible Newtonian fluids mixtures. ${ }^{7}$ According to the observation on immiscible
Newtonian fluids mixtures ${ }^{7}$ under shear flow with a video-microscope, the domains are elongated with increasing shear rate, followed by aggregation when they touch each other, and after a while, the long domains are ruptured to the shorter ones. These process may be a reason why there exist the undershoot and overshoot for shear stress and primary normal stress difference, respectively, when shear rate is increased. At steady state, the diameters of domains become rather uniform and are inversly proportional to shear rate, though there is a distribution in the length of domains.

More detailed discussion on the relationship between the structure change and viscoelastic properties of immiscible polymer blends will be reported in near future. The reason for break down of the scaling relations at high shear rate $\left(\dot{\gamma}>\dot{\gamma}^{*}\right)$ for polymer blends may be clarified by observing the structure change.

Acknowledgements. We wish to thank Professor M. Doi of Nagoya University for valuable discussions. This work was supported by a Grant-in-Aid for Scientific Research (No. 04453105) from the Ministry of Education, Science, and Culture of Japan.

\section{REFERENCES}

1. L. A. Utracki, "Polymer Alloys and Blends," Hanser Publishers, Munich, 1989.

2. Y. Takahashi, H. Suzuki, Y. Nakagawa, I. Noda, Polymer International, 34, 327 (1994).

3. M. Doi and T. Ohta, J. Chem. Phys., 95, 1242 (1991).

4. M. Doi, in "Lecture Notes in Physics," L. Garrido, Ed., Springer-Verlag, Berlin, 1993.

5. R. G. Larson and D. W. Mead, J. Rheol., 33, 1251 (1989).

6. R. G. Larson and M. Doi, J. Rheol., 35, 539 (1991).

7. Y. Takahashi, N. Kurashima, I. Noda, and M. Doi, J. Rheol, 38, 699 (1994). 